\title{
Experience-Dependent Plasticity Is Impaired in Adult Rat Barrel Cortex after Whiskers Are Unused in Early Postnatal Life
}

\author{
V. Rema, ${ }^{1}$ Michael Armstrong-James, ${ }^{1,2}$ and Ford F. Ebner ${ }^{1}$ \\ ${ }^{1}$ Department of Psychology, Vanderbilt University, Nashville, Tennessee 37240, and 2Department of Neuroscience, Queen Mary Westfield College, \\ University of London, London E1 4NS, United Kingdom
}

The capacity of adult barrel cortex to show experience-dependent plasticity after early restricted neonatal sensory deprivation was analyzed in barrel field cortex neurons. Selective sensory deprivation was induced by trimming two whiskers from postnatal day 0 (P0) to P21, namely, the principal D2 whisker plus one adjacent surround whisker (D3). At maturity (P90), responses of supragranular (layer II/III) and barrel (layer IV) neurons, all located in the D2 barrel column, were analyzed for modified responses to the deprived principal whisker (D2) and the nondeprived (D1) and deprived (D3) adjacent surround whiskers. For supragranular neurons, the responses to both principal and surround whiskers were reduced at maturity, whereas the barrel neurons showed mildly elevated responses to the principal whisker but a reduced response to the deprived surround whisker. In normal adult rats, trimming all but the principal D2 whisker and an adjacent D3 whisker for $3 \mathrm{~d}$ (whisker pairing) produced the expected bias: elevated responses from the intact D3 compared with the cut D1 whisker in both barrel and supragranular neurons. When the neonatally deprived D2 and D3 whiskers were paired at maturity, a similar D3/D1 bias was generated in barrel neurons, but no bias occurred in supragranular neuron responses. Pairing the maintained D1 and deprived D2 whiskers produced a much greater bias toward D1 compared with the deprived D3 whisker in barrel neurons than in supragranular neurons. There were minimal effects on response latencies in layer IV under any of the experimental conditions. These findings indicate that a restricted period of sensory deprivation in early postnatal life (1) impairs intracortical relay of deprived inputs from layer IV to layer II/III in barrel cortex at maturity and (2) degrades receptive field plasticity of the supragranular layer cells but not the thalamic-recipient barrel neurons.

Key words: neural plasticity; barrel field cortex; rat; sensory deprivation; cortical development; whisker pairing

\section{Introduction}

Weisel and Hubel (1965) showed that restricted monocular visual experience during a critical postnatal period leads to degraded visual cortex responses to the deprived eye, and that these responses are still apparent after kittens or monkeys mature. Similar experiments at maturity failed to modify receptive fields. However, in the somatosensory system it is now well established that plasticity to peripheral nerve damage in the primary somatosensory (SI) cortex can be achieved in adult animals (Kalaska and Pomeranz, 1979; Rasmusson, 1982; Merzenich et al., 1983). More recently, it has been shown that simple changes in behavioral use can modify SI cortical representations. For example, behavioral tactile training expands the relevant SI cortical columns in monkeys (Clark et al., 1988; Recanzone et al., 1992a,b), and the altered use of selected whiskers in adult rats potentiates barrel cortical neuron responses to those whiskers (Diamond et al., 1993; Armstrong-James et al., 1994). In adult rats, the effect of deprivation is maximal for the cortical columns that receive low levels

\footnotetext{
Received Aug. 29, 2002; revised Sept. 19, 2002; accepted 0ct. 25, 2002.

This study was supported by National Institutes of Health Grants NS-25907 and NS-13031. General support was provided by the Vanderbilt University Vision Research Center and the John F. Kennedy Center. We thank Anita Sankaran for expert assistance with histology.

Correspondence should be addressed to Ford Ebner, Department of Psychology, 301 Wilson Hall, 11121st Avenue South, Vanderbilt University, Nashville, TN 37240. E-mail: ford.ebner@vanderbilt.edu.

Copyright $\odot 2002$ Society for Neuroscience $\quad$ 2270-6474/02/220358-09\$15.00/0
}

of activity from the deprived periphery (Simons and Land, 1987; Wallace et al., 2001).

Because it is now established that the SI cortex exhibits continued cortical plasticity throughout life, we were interested in the influence of early sensory deprivation on the subsequent plasticity of SI cortical neurons after the animals grow to maturity. We ask two questions in this study: (1) Does sensory deprivation of only two neighboring tactile inputs (whiskers) in neonatal rats produce deficits in sensory processing restricted to their target barrel columns at maturity? (2) Do the neonatally deprived inputs exhibit specific failures to generate barrel-column plasticity to novel sensory experience at maturity? We report that trimming two adjacent whiskers from birth for $21 \mathrm{~d}$ compromises the responses of supragranular neurons (layer II/III), but not layer IV neurons, to these whiskers at maturity. In addition, paired use of the neonatally trimmed whiskers at maturity fails to induce sensory plasticity in supragranular neurons, whereas barrel neurons retain their ability to modify responses to this novel experience.

\section{Materials and Methods}

All animals were male Long-Evans rats bred in-house. All experimental procedures were approved by the Vanderbilt University Committee on Animal Use in accordance with National Institutes of Health guidelines.

Experimental groups. The experimental design required comparisons of neurotransmission for neurons in the D2 barrel (layer IV) and supragranular (layer II/III) layers above the D2 barrel in the SI cortex. Thus, 


\section{A. Normal}

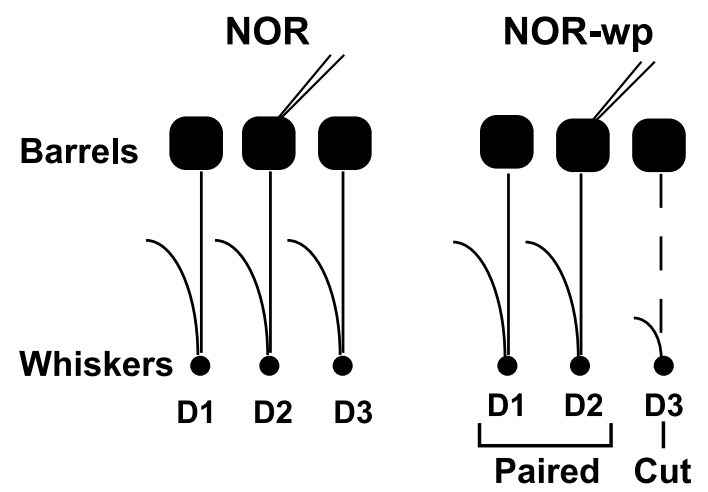

\section{B. Neonatally deprived}
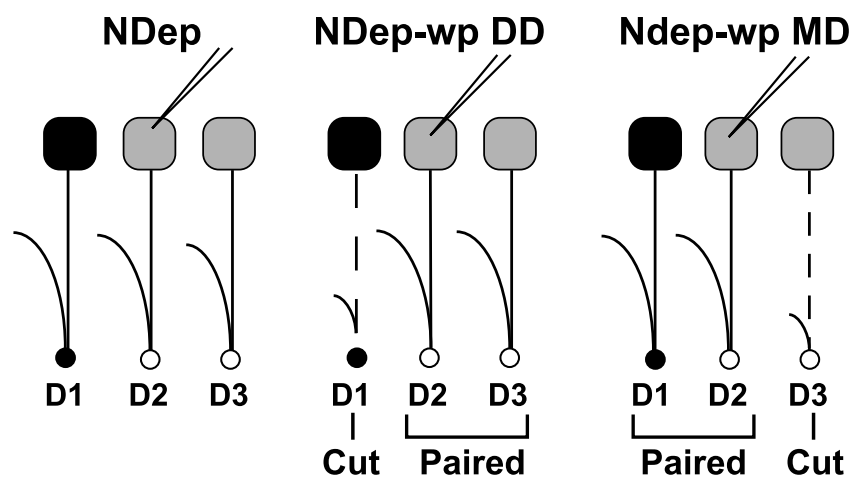

Figure 1. Schematic diagrams illustrating the manner in which whiskers were trimmed for early deprivation and adult whisker pairing in the various experimental groups. $A$, For normal adult animals (3 months of age), responses of single neurons in the D2 barrel column were documented for response to their principal D2 and surround D1 and D3 whiskers (NOR). In a second group, all whiskers except D2 and D1 or D3 were trimmed for $3 \mathrm{~d}$ (whisker-pairing), and $\mathrm{D} 2$ neurons were tested for responses to D1, D2, and D3 whisker stimulation (NOR-wp). B, For all three groups subjected to neonatal deprivation (NDep, NDep-wp-DD, and NDep-wp-MD), only the D2 and D3 whiskers (open circles) were trimmed every day from P0 to P21. Physiological responses were tested at maturity (3 months of age). NDep, Early deprived animals not subjected to adult whisker pairing. NDep-wp DD, Animals had both early deprived whiskers (D2 and D3) acutely whisker paired as adults. NDep-wp MD, Animals had the neonatally deprived D2 whisker paired with the neonatally intact (maintained) D1 whisker. The important distinction between NDep-wp-MD and NDep-wp-DD is that the surround whisker paired in the adult transmits from a deprived barrel in one case and from a nondeprived barrel in the other case to test their ability to activate cells in the deprived D2 barrel column. See Materials and Methods for details.

recordings were restricted to D2 barrel-column neurons in all animals. Measurements were made in normally reared rats and in rats with early sensory deprivation [from postnatal day 0 (P0) to P21]. Five groups of five to eight animals were prepared on the basis of the animal's previous experience of whiskers D1, D2, and D3 before recording (Fig. 1). These groups were as follows. (1) NOR indicates normal adult animals with all whiskers always intact. (2) NOR-wp indicates normal adult whiskerpaired (WP) animals, meaning that all except the principal whisker (D2) and an in-row adjacent whisker (D1 or D3) on the right side of the face were clipped to the level of the fur for $3 \mathrm{~d}$ in adult rats; all whiskers on the left side of the face remained intact. During the $3 \mathrm{~d}$, the spared whiskers were used for tactile exploration while the surrounding barrel columns were inactive. Receptive-field analysis under urethane anesthesia was conducted $3 \mathrm{~d}$ after the onset of whisker trimming. (3) NDep indicates adult animals with a history of neonatal trimming of whiskers D2 and D3 on the right side of the face to the level of the fur every day from P0 to P21.
These animals were analyzed without WP as adults. (4) NDep-wp-DD indicates animals whose deprived whiskers (D2 and D3) received $3 \mathrm{~d}$ of WP experience at maturity (DD indicates that both deprived whiskers were paired). (5) NDep-wp-MD indicates rats whose maintained (nondeprived) D1 plus the neonatally deprived principal D2 whisker were WP for $3 \mathrm{~d}$ at maturity (MD indicates maintained/deprived pairing).

Experimental animals lived in standard plastic rat cages with two normal littermates during the $3 \mathrm{~d}$ between whisker clipping and the onset of physiological recording. Whisker-trimmed animals appeared to move around the cage in a normal manner.

Anesthesia and surgery. All animals were anesthetized with urethane (1.5 gm/kg body weight, i.p.; $30 \%$ solution in water). Core body temperature was maintained at $36.5^{\circ} \mathrm{C}$ by a rectal thermistor controlling a heating pad through an electronic interface. A bone opening was made from 4 to $7 \mathrm{~mm}$ lateral to the midline and from 0 to $4 \mathrm{~mm}$ posterior to bregma to expose the barrel field cortex. Because anesthetic level profoundly affects response characteristics (Armstrong-James and George, 1988; Friedberg et al., 1999), anesthesia was maintained by supplemental urethane injections ( $10 \%$ of the original dose) such that layer $\mathrm{V}$ neurons, sampled at the end of each penetration, exhibited bursts that were characteristic of stage III slow-wave sleep and light anesthesia (2-4 bursts/ sec) (Armstrong-James et al., 1985; Fox and Armstrong-James, 1986). This condition enabled rats to be maintained in full but light anesthesia with no signs of discomfort. Under these conditions, long-latency and sluggish hindlimb withdrawal typically was achieved with firm pinching of the hindpaw, respiration was regular (within $80-110$ breaths/min in nearly all animals), and there was no overt whisking, spontaneous movement, or whisker tremor.

Single-neuron electrophysiological recording. Single-unit recording was performed using carbon fiber microelectrodes (Armstrong-James and Millar, 1979). Single neurons were discriminated using a BAK waveform/ window discriminator (BAK Electronics, Germantown, MD). All penetrations were radial, perpendicular to the cortical surface, through a very small slit in the dura and targeted on the D2 barrel column. Only neurons responding maximally and at the shortest latency to the $\mathrm{D} 2$ whisker were analyzed. Microelectrodes were advanced with three-dimensional microdrives with an accuracy of $5 \mu \mathrm{m}$ in all three dimensions. The positions of all penetrations were mapped together with the subpial depths of neurons. Traversing the subarachnoid space was examined optically by watching the black tip of the microelectrode contact the surface of cortex, and contact was heralded with an increase of noise by the electrode $(\sim 50 \%)$. During microelectrode egress, neuron positions were invariably accurate to within $25 \mu \mathrm{m}$. Consistent with previous studies (Armstrong-James et al., 1994), alternate penetrations were marked by a $5 \mathrm{sec}, 2.5 \mu \mathrm{A}$ tip-negative current that enabled locations of unmarked penetrations to be interpolated within $\sim 5 \mu \mathrm{m}$ by geometric reconstruction (see below).

Whisker stimulation. Cortical cell responses were recorded to individual stimulation of the D1, D2, and D3 whiskers. Each whisker was cut to the same length of $4-5 \mathrm{~mm}$ so that they could be stimulated at the same distance from the base. The whiskers on the right side of the face were stimulated using a thin wooden rod glued to one end of a piezoelectric "bimorph" ceramic wafer positioned just behind the whisker, $4-5 \mathrm{~mm}$ from the skin. The wafer was deflected by a computer-gated current producing a $300 \mu \mathrm{m}$ trapezoidal anterior-to-posterior movement of the free end with rise and fall times of $0.5 \mathrm{msec}$ and a total stimulus duration of $3 \mathrm{msec}$. Fifty stimuli were used for each block of trials delivered at $1 \mathrm{~Hz}$. For each cell, one block of stimulus trials was presented to whisker D2 and then to each of its immediate neighbors (D1 and D3).

Data collection and analysis. Data collection was programmed with a PC computer (Dell Computer Company, Round Rock, TX) driving a 1401plus CED interface (Cambridge Electronic Design, Cambridge, UK). All raw data on the timing of action potentials were stored off-line for additional analysis. Poststimulus time histograms (PSTHs) were constructed from $1 \mathrm{msec}$ bins, where bin $0-1$ was registered as the first bin after stimulus (latency $0-1 \mathrm{msec}$ ). Latency histograms (LHs) were constructed for all neurons using the time bin of the first action potential generated by each stimulus. Latencies cited in the text refer to modal latencies in which the modal latency is defined as the time of the most 


\section{Barrel Neurons}
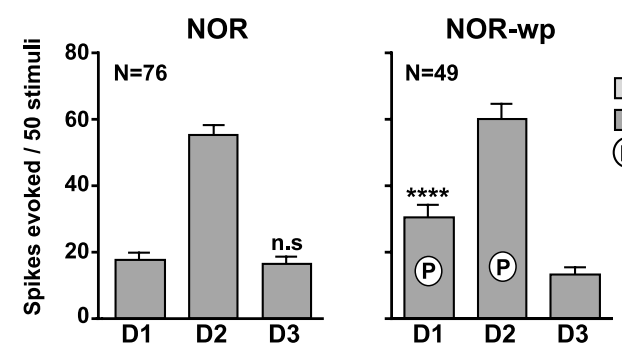

Whisker Stimulated
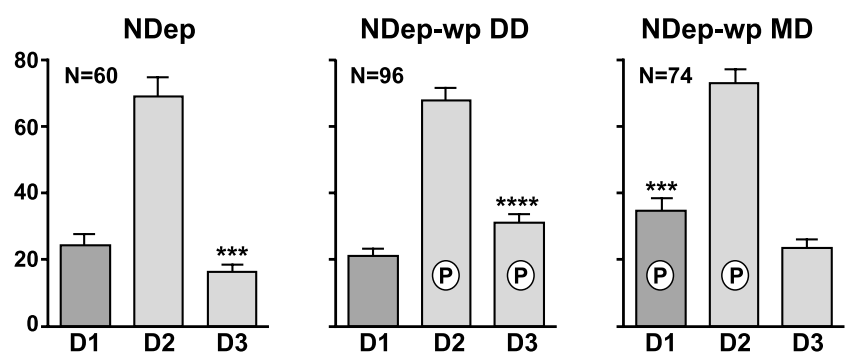

Figure 2. Response magnitudes (means \pm SEM) for neurons located in the D2 barrel in response to individual stimulation of three adjacent whiskers for the five groups of adult rats. Spike counts for each neuron were derived from $100 \mathrm{msec}$ PSTHs as a result of 50 stimuli applied to one of the D1, D2, or D3 whiskers. Whisker D2 is always the principal whisker, and D1 and D3 are always the adjacent in-row surround whiskers. All data are from neurons histologically confirmed to be in the D2 barrel. For calculation of response magnitude, see Materials and Methods. NOR, Normal rats that had all whiskers maintained intact. NOR-wp, Normal rats that at maturity ( $90 \mathrm{~d}$ of age) were subjected to $3 \mathrm{~d}$ of whisker pairing of the D1 surround and D2 principal whiskers (see Materials and Methods). $P$ on bars indicates the whiskers that were acutely paired at maturity in this and all subsequent figures. Bottom row, Results for neonatally deprived rats that had two whiskers (D2 and D3) cut (deprived) from P0 to P21. Note that bars for deprived whiskers are gray. NDep, Results from neonatally deprived animals without adult whisker-pairing experience at maturity. NDep-wp DD, Results from neonatally deprived animals in which two deprived whiskers (D2 and D3) were acutely whisker paired at maturity. NDep-wp $M D$, Results from animals in which neonatally deprived D2 and neonatally maintained D1 whiskers were paired at maturity. $x$-axis, Whiskers stimulated. $y$-axis, Response magnitude cast as spikes per 50 stimuli. Response magnitudes for surround whiskers D1 and D3 are assessed for statistical differences within each individual group $\left({ }^{* *} p<0.001\right.$; ${ }^{* * *} p<0.0001$; Wilcoxon signed-rank test). n.s, Not significant. Error bars indicate SEM. N, Number of neurons from five to eight animals in each group.

common first spike (bin) after stimulus produced by the 50 stimuli (Armstrong-James and Fox, 1987).

Analysis of PSTHs. Response magnitudes were quantified by cumulative counts of spikes generated in PSTHs during periods from 0 to 100 msec after stimulus. Counts were corrected for spontaneous activity by subtracting the average number of spontaneous events per $1 \mathrm{msec}$ bin occurring $100 \mathrm{msec}$ before stimulus. The mean count was subtracted from each response after adjustment pro rata for the $100 \mathrm{msec}$ poststimulus period. Statistical analysis was performed on exported data using nonparametric tests, either the Mann-Whitney $U$ test (MWU) or the Wilcoxon signed-rank test with StatWorks software (Cricket Software, Malvern, PA). Only data from neurons histologically proven to be in the D2 barrel column (see below) are included in the results.

Histology. After termination of the experiment, rats were deeply anesthetized with urethane and perfused with $0.1 \mathrm{M}$ PBS followed by $4 \%$ buffered paraformaldehyde. After the brain was immersed overnight in $10 \%$ sucrose in PBS, the neocortex was separated from the underlying structures, gently flattened between glass slides, and then transferred to $20 \%$ sucrose in PBS for $1 \mathrm{~d}$. Tangential sections were cut at $40 \mu \mathrm{m}$ and processed for cytochrome oxidase activity (Wong-Riley and Welt, 1980). Locations of recording sites were reconstructed from the microlesions to

\section{Supragranular Neurons}

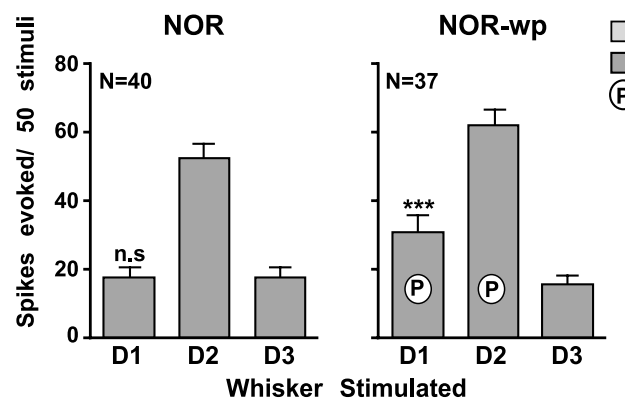

Deprived whisker

Maintained whisker

(P) Paired whisker

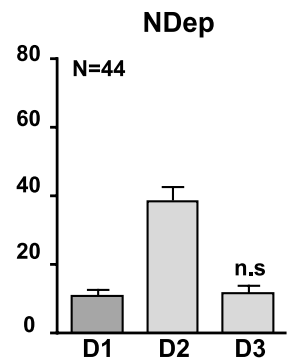

NDep-wp DD

NDep-wp MD
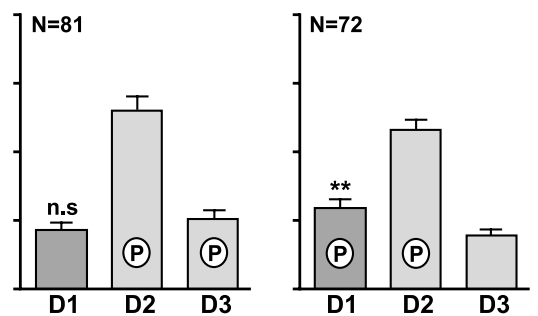

Figure 3. Mean response magnitudes for neurons located in the supragranular layers immediately above the D2 barrel to the stimulation of whiskers D1, D2, and D3 for the five groups of adult rats. All data are from neurons in the same penetrations used to collect the barrel neuron data in Figure 2. NOR, Results for normal rats with all whiskers intact. NOR-wp, Results from normal rats acutely whisker paired for $3 \mathrm{~d}$ in which D1 and D2 whiskers were paired, as indicated by P. Bottom row, Results for neonatally deprived rats that had two whiskers (D2 and D3) cut from P0 to P21. Note that bars for deprived whiskers are gray.NDep, Neonatally deprived animals without whisker-pairing experience. NDep-wp $D D$, Neonatally deprived animals in which two deprived whiskers (D2 and D3) were acutely whisker paired as above. NDep-wp MD, Animals in which neonatally deprived $D 2$ and neonatally maintained $D 1$ whiskers were whisker paired at maturity. ${ }^{* *} p<0.01 ;{ }^{* * *} p<0.001$. n.s, Not significant. For additional details, see Figure 2.

ascertain that the recorded neurons were within the D2 barrel. A neuron was considered to be within barrel D2 if the recording site was localized within the vertical and horizontal bounds of barrel D2 in the reconstruction of cytochrome oxidase-stained tangential sections. Only neurons located within the D2 barrel column were used for this study. No neurons located in the septa between barrels or other barrel territories were included in our analysis.

\section{Results}

Deprivation-based alterations in receptive fields:

barrel (layer IV) neurons

Figure 2 shows the mean response magnitudes of neurons in the D2 layer IV barrel to stimulation of the D-row center (D2) and surround D1 and D3 whiskers for the five groups of animals. The population of normal rat D2 barrel neurons (Fig. 2, NOR) responds with equivalent response magnitudes to both D1 and D3 surround whiskers $[p>0.8$; Wilcoxon matched-pair rank test (WMPR)]. After $3 \mathrm{~d}$ of D1 and D2 WP (Fig. 2, NOR-wp), the D2 barrel neuron population shows a highly significant bias toward the paired D1 whisker compared with the unpaired D3 whisker $(p<0.0001$; WMPR).

For the deprived conditions (Fig. 2, bottom graphs), D2 barrelcolumn neuron plasticity is examined at maturity after neonatal cutting of D2 and D3 whiskers from P0 to P21. All whiskers were allowed to regrow (see Materials and Methods) between P21 and P90. In neonatally deprived rats that received no subsequent WP (Fig. 2, NDep), the deprived D3 whisker produces significantly 


\section{Barrel Surround Whisker Ratios}
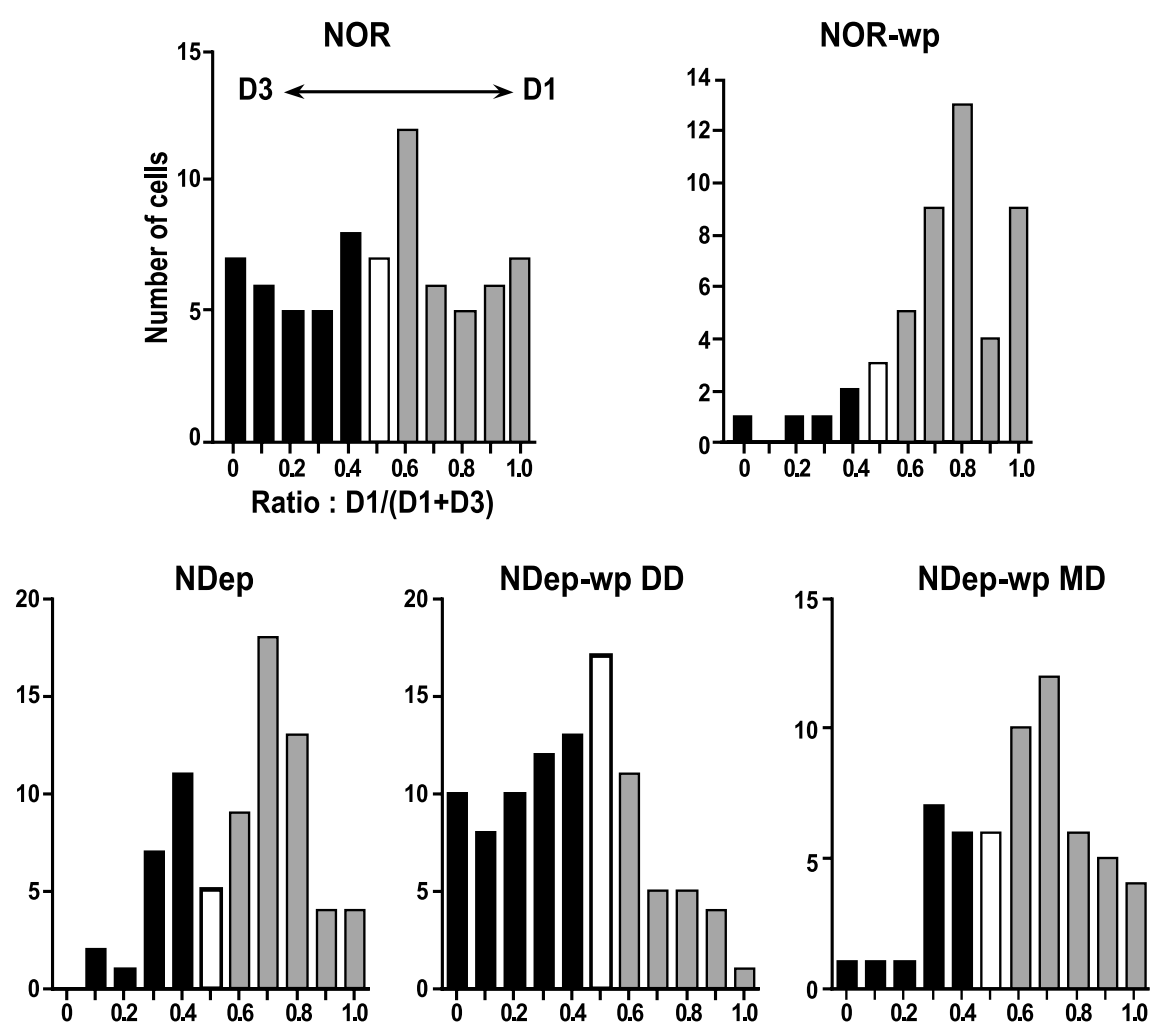

Figure 4. Cell-by-cell response bias to D-row surround whiskers D1 or D3 adjacent to the D2 whisker for neurons located only in the barrel of the D2 barrel column. Bias ratio is measured by dividing the D1 response magnitude by the sum of D1 and D3 response magnitudes for each neuron. Neurons responding only to D1 are given a value of 1 , and neurons responding only to D3 are given a value of 0 . Equal responses generate a value of 0.5 . Neurons responding best to D3 or D1 are reflected by black or gray bars in histograms, respectively. Equal responses are indicated by white bars. Surround whisker bias is compared for normal (NOR) and WP normal (NOR-wp) rats. For the latter, the D1 whisker was paired at maturity with the D2 whisker. Surround whisker bias is shown for neonatally deprived rats without whisker pairing (NDep), with whisker pairing of neonatally deprived D2 and D3 whiskers (NDep-wp DD), and with whisker pairing of the neonatally deprived D2 and maintained surround D1 whisker (NDep-wp MD).

reduced responses in D2 barrel cells compared with the maintained D1 whisker ( $p<0.005$; WMPR). That is, neonatal deprivation of the D3 whisker chronically diminished the response to the D3 whisker from D2 barrel cells.

To examine the status of experience-dependent plasticity in barrel neurons in adult rats with neonatally deprived D2 and D3 inputs, these deprived whiskers (D2 and D3) were WP for $3 \mathrm{~d}$ at maturity (Fig. 2, NDep-wp DD). These conditions resulted in a highly significant increase in the response of barrel neurons to the paired surround D3 whisker compared with the untrimmed D1 whisker $(p<0.0001$; WMPR). Therefore, although the depressed, deprived inputs were reduced in their ability to drive cortical cells, they could be upregulated in their effect on D2 barrel cells when challenged by WP.

If the postnatally nondeprived (maintained) D1 whisker was WP with the deprived principal D2 whisker in the adult (Fig. 2, NDep-wp MD), then the response to the maintained D1 whisker was increased significantly compared with the cut D3 whisker $(p<0.005$; WMPR).

\section{Alterations in receptive fields: supragranular (layer} II/III) neurons

Figure 3 shows mean response magnitudes for the five experimental groups of animals for neurons in the supragranular layers in the D2 column. These neurons were encountered above the barrel neurons in the same penetrations as the neurons in Figure 2. Like the barrel neurons in normal rats, the supragranular neurons of the D2 barrel column exhibited equivalent response magnitudes to D1 and D3 surround whiskers (Fig. 3, NOR). Differences in magnitude to D1 and D3 surround whiskers were not significant ( $p>0.8$; WMPR). After $3 \mathrm{~d}$ of D1 and D2 WP in normal rats (Fig. 3, NOR-wp), a highly significant bias occurred toward the paired D1 whisker compared with the unpaired D3 whisker $(p<$ 0.005; WMPR), which was similar to activity-initiated changes in barrel neuron responses.

In the neonatally deprived adult rats without any acute WP (Fig. 3, NDep), no significant D1/D3 bias occurred in the response magnitude to the neonatally intact D1 whisker in the supragranular neurons ( $p>0.8$; WMPR). Therefore, in contrast to barrel neurons, neonatal deprivation of the D3 whisker did not cause a reduced response compared with the maintained D1 whisker in layer II/III. However, neonatal deprivation resulted in reduced responses to both D1 and D3 surround whiskers compared with normal rats $(p<0.03$ for D1 and D3; MWU). Response magnitude to the neonatally trimmed principal whisker D2 was also lower than that for the normal rats ( $p=0.03$; MWU).

When the neonatally deprived D2 and D3 inputs are paired for $3 \mathrm{~d}$ at maturity (Fig. 3, NDep-wp DD), supragranular layers failed to show an increase in response to the paired surround D3 whisker $(p>0.1$ for D1 - D3 differences; WMPR) in contrast to barrel neurons.

Finally, acutely pairing the neonatally nondeprived D1 with the neonatally deprived D2 whiskers (Fig. 3, NDep-wp MD) resulted in a small but significant increase in response to the maintained D1 whisker compared with the neonatally deprived D3 whisker $(p<0.01$; WMPR).

Therefore, the results show the following: (1) when the D2 barrel column is deprived of its center and one adjacent surround whisker input during the early postnatal period of SI cortex development, the mature barrel neurons develop a bias toward the intact surround whisker at maturity, whereas supragranular neurons fail to develop this bias; and (2) after WP, the deprived inputs in the neonatally deprived rats successfully generate a bias in response to the paired deprived surround whisker for barrel neurons, similar to normal rats, but fail to generate any bias to the paired deprived whisker in supragranular neurons.

\section{Neuron-by-neuron surround bias}

Barrel neurons

Figure 4 shows the relative bias of responses toward D1 or D3 whiskers displayed on a neuron-by-neuron basis for barrel neurons in the five groups of animals. In normal animals (Fig. 4, $N O R$ ), in which no WP was performed, no bias is evident for barrel neurons to respond best to either the D1 or the D3 sur- 
round row whiskers ( $p>0.9$; WMPR). However, 3 d of WP of D1 and D2 whiskers in normal animals (Fig. 4, NOR-wp) generates a strong bias in most D2 barrel neurons toward the paired D-row whisker D1 $(p<0.0001$; WMPR). In the neonatally deprived animals (Fig. 4, NDep), a substantial percentage of neurons respond better to the preserved surround whisker D1 and respond less well to the neonatally deprived whisker D3 ( $p<0.004$; WMPR). When the neonatally deprived D2 and D3 whiskers are acutely WP (Fig. 4, NDep-wp $D D)$, a successful bias is generated toward the deprived D3 whisker and away from the paired D3 whisker $(p<0.001$; WMPR). Similarly, when the preserved D1 whisker is paired with the deprived D2 principal whisker (Fig. 3, NDep-wp MD), a strong bias develops toward D1 and away from D3 ( $p<0.001$; WMPR). These findings are in agreement with and add weight to the statistical findings for mean differences in response magnitudes to surround whiskers in the different conditions (Fig. 2).

\section{Supragranular neurons}

Figure 5 shows the relative bias of responses toward either the paired or the cut whisker on a neuron-by-neuron basis for supragranular layers. Neurons were in the same penetrations as the barrel neurons described above for the five groups of animals. In the normal animals (Fig. 5, NOR), no bias is evident for either D1 or D3 whisker ( $p>0.9$; WMPR). However, acute pairing of the D1 and D2 whiskers (Fig. 5, NOR$w p$ ) generates a profound bias toward the paired whisker D1 ( $p>0.005$; WMPR). In contrast to the finding for barrel neurons, the supragranular neurons of neonatally deprived animals (Fig. 5, NDep) show no bias toward either surround whisker $(p>$ 0.8 ; WMPR). After $3 \mathrm{~d}$ of WP of the neonatally deprived D2 and D3 whiskers (Fig. 5, NDep-wp DD), no significant bias occurs toward the paired neonatally deprived D3 whisker $(p>0.1$; WMPR). However, when the neonatally preserved D1 whisker is paired with the neonatally deprived D2 center whisker (Fig. 5, NDep-wp MD), a small bias toward D1 and away from D3 is generated $(p>0.01$; WMPR).

Principal whisker (D2) transmission from barrel to supragranular layers

On the basis of latency measurements, there is strong evidence that a majority of neurons in layer II/III in barrel cortex do not fire action potentials monosynaptically in response to thalamic afferents (Armstrong-James, 1995). Therefore, the discharge of supragranular neurons in the D2 barrel column to D2 whisker inputs overwhelmingly depends on relay from layer IV neurons. Consequently, the efficacy of sensory transmission from layer IV to superficial layers should be reflected in the response magnitudes to the principal D2 whisker input in barrel neurons compared with responses in supragranular neurons in the same col-

\section{Supragranular Surround Whisker Ratios}

NOR
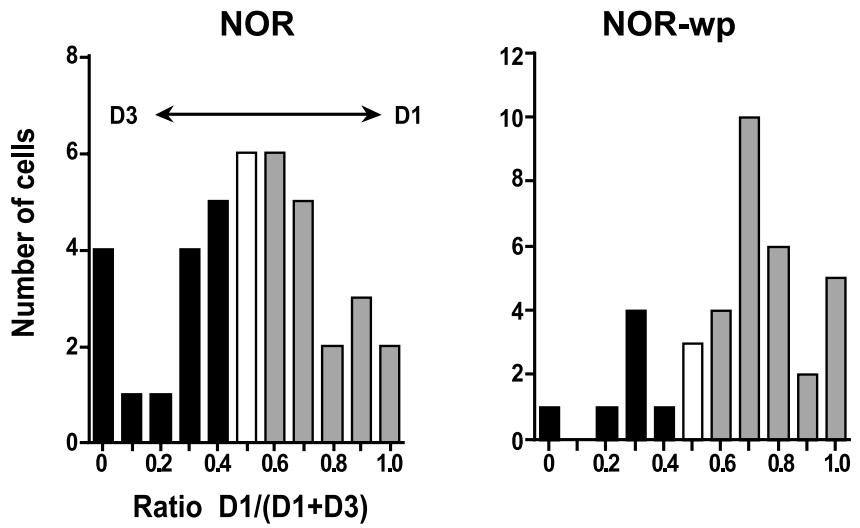

Ratio D1/(D1+D3)
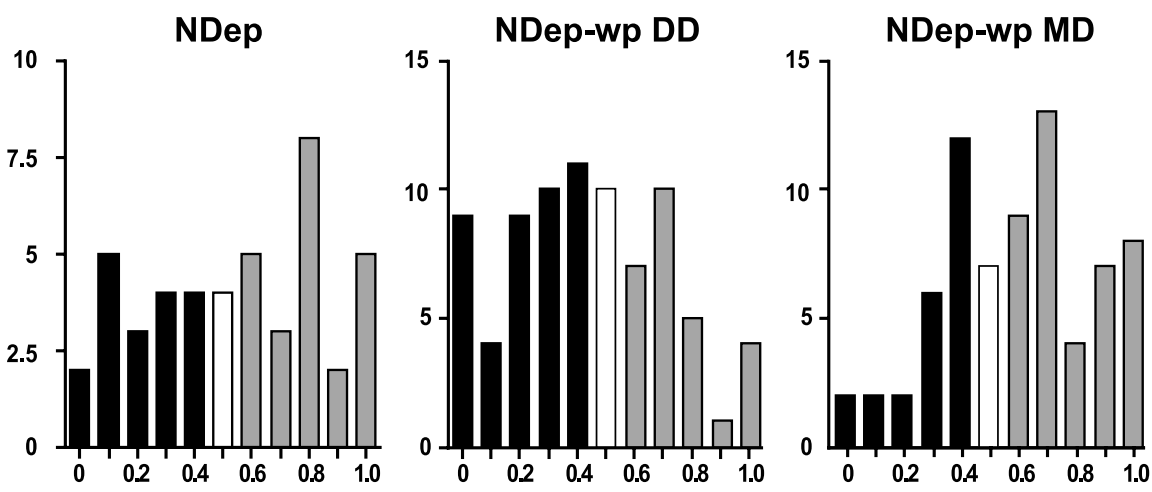

Figure 5. Cell-by cell response bias to D-row surround whiskers D1 or D3 adjacent to the D2 whisker for neurons located only in the supragranular layers of the D2 barrel column. Neurons were encountered in the same penetrations as barrel neurons described in Figure 4. Nomenclature, histogram arrangements, and other details are as in Figure 4.

\section{Principal Whisker Transmission}

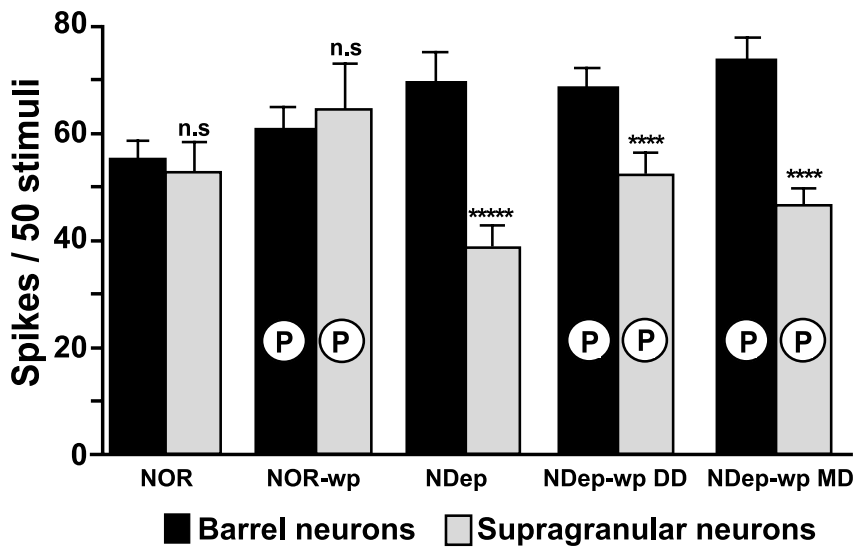

Figure 6. Statistical comparison of $\mathrm{D} 2$ barrel-column response magnitudes to stimulation of the D2 principal whisker for the five groups of rats investigated. The data are derived from those shown in Figures 2 and 3. $y$-axis, Response magnitude. $x$-axis, Experimental group. Here a paired whisker $(P)$ is a D2 whisker that was paired with either D1 or D3 during acute whisker pairing at maturity. Response magnitudes to the principal whisker in barrel and supragranular locations do not differ statistically for normal rats regardless of whether they were (NOR-wp) or were not (NOR) subjected to whisker pairing (MWU). For neonatally deprived animals (NDep, NDep-wp DD, and NDep-wp MD), responses in the supragranular layers were significantly lower than for neurons in barrel locations in the same penetrations (MWU). ${ }^{* * *} p<0.0001$; ${ }^{* * * * *} p<0.000001$. n.s, Not significant. For additional explanation of terms, see the legend to Figure 2. 


\section{Transmission of Surrounds}

\section{A NORMAL (NOR)}

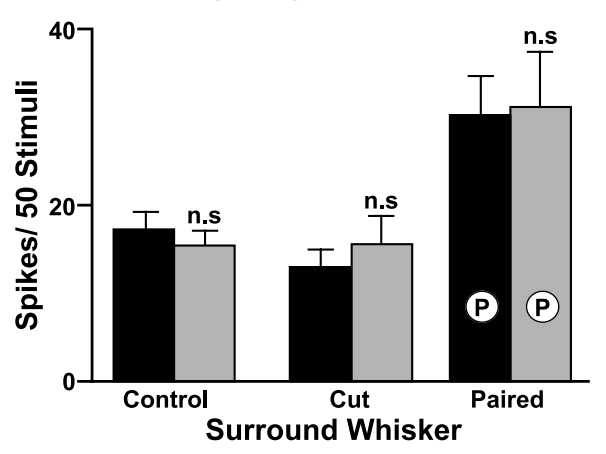

$\square$ Supragranular neurons

Barrel neurons

(P) Paired whisker

\section{B NEONATALLY DEPRIVED}

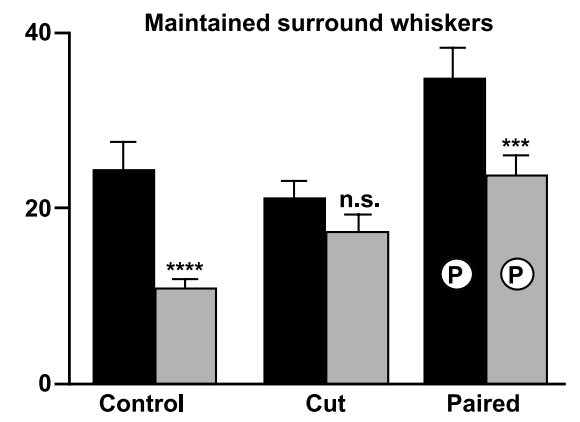

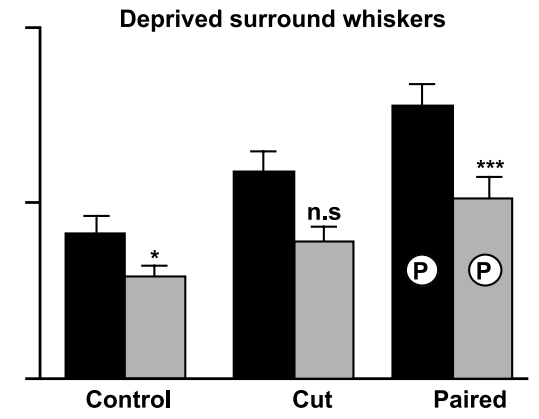

Figure 7. Statistical comparison of response magnitudes to the surround D-row (D1 or D3) whisker in supragranular (gray bars) and barrel (black bars) layers for the five groups of rats investigated. The data are derived from Figures 3 and 5 . Asterisks signify levels of statistical significance as in Figure 2, but using the MWU. $y$-axis, Response magnitude as spikes per 50 stimuli. $x$-axis, Experimental group. Control surround whiskers are those from rats not subjected to whisker pairing. A paired whisker is a D1 or D3 surround whisker that was paired with the D2 whisker during acute whisker pairing at maturity ( $P$ on histograms). Cut whiskers are those cut for whisker pairing at adult age. $A$, Response magnitudes for equivalent whiskers do not differ statistically between barrel and supragranular locations for normal rats with or without whisker pairing (MWU). B, Responses in the supragranular layers are smaller than for neurons in barrel locations in the same penetrations for control and paired whiskers, and the responses are reduced, but not significantly, for cut whiskers (MWU). ${ }^{*} p<0.05$; $^{* * *} p<0.001$; $^{* * * *} p<0.0001$. n.s, Not significant. For additional explanation of terms, see Figure 6.

umn. The efficacy of this transmission is compared for the various groups in Figure 6.

Figure 6 compares response magnitudes produced by the principal D2 whisker in D2 barrel neurons (black bars) and in D2 supragranular neurons (gray bars). Statistical differences in response magnitude to stimulation of the same D2 whisker for supragranular and barrel neurons show a profound effect of neonatal sensory deprivation on supragranular neurons that is not present in barrel neurons. For normally reared animals, responses of supragranular neurons were not significantly different from those for the barrel neuron population ( $p>0.3$; MWU). WP for $3 \mathrm{~d}$ increases the response of supragranular neurons to D2 and increases the response of barrel neurons ( $p>0.3$; MWU). For all conditions in which neonatal deprivation of D2 and D3 whiskers was used, responses to the deprived D2 whisker were profoundly lower for supragranular neurons compared with barrel neurons ( $p<0.001$ for NDep, NDep-wp-DD, and NDep-wp$\mathrm{MD}$; MWU). For transmission of D2 whisker activation from layer IV to layer II/III, the most profound decrement is observed in non-WP animals (NDep difference for D2 response magnitudes, $p<0.0001$; MWU).
Surround D-row whisker transmission from barrels to supragranular layers Figure 7 compares response magnitudes for surround whisker inputs to barrel and supragranular layers under normal and neonatally deprived conditions. In Figure $7 A$, surround whisker responses are compared between barrel and supragranular locations for control (non-WP) and WP animals.

Responses of supragranular neurons were not significantly different from responses for barrel neurons for any surround whisker type under any condition ( $p>0.1$ for control, paired, and cut; MWU). WP essentially doubled the response magnitudes to the paired surround whisker compared with controls or to cut whiskers in both barrel and supragranular neuron populations (Fig. 7A).

In Figure $7 B$, surround whisker responses are compared between barrel and supragranular locations for all neonatally deprived conditions. Whisker types are grouped into those that were trimmed neonatally (deprived) and those that were not trimmed (nondeprived or maintained) during the first 3 weeks of life. For maintained surround whiskers, response magnitudes were smaller in the supragranular layers than in the barrel for both control and WP conditions $(p<0.001$ and $p<$ 0.005 , respectively). For the cut whisker in the WP condition, responses were not significantly different in supragranular and barrel neuron populations $(p>0.05)$. For neonatally deprived whiskers, cortical responses to both the control and the paired whiskers were significantly smaller in the supragranular layers than for the same whiskers in the barrel layer $(p<0.05$ and $p<0.005$ for control and for paired, respectively). Cut whisker response differences between supragranular and barrel neurons were not significant $(p>0.05)$. In contrast to normally reared rats (Fig. $7 A$ ), response magnitudes for all neonatally deprived whiskers (Fig. $7 B$ ) were smaller for the supragranular neuron population than for barrel neurons.

\section{Deprivation-related latency changes}

In the barrel, the D2 principal whisker latencies under all conditions exhibited rather similar distributions (Fig. 8, left), and $\sim 60 \%$ of latencies were $<10$ msec. Approximately $98.5 \%$ of latencies were $<15 \mathrm{msec}$ in barrels of normal rats, falling marginally to $91.7 \%$ with pairing. In NDep rats, $92 \%$ of latencies were $<15$ msec. With pairing, the $<15 \mathrm{msec}$ latencies in barrels changed only marginally to $88.3 \%$ for NDep-wp-DD and $94.6 \%$ for NDep-wp-MD. However, differences in latencies between barrel cells in normal rats (Fig. 8, NOR) and all other conditions (NOR-wp, NDep, NDep-wp MD, and NDep-wp DD) were not significant $(p<0.2$ in all instances; MWU). The data suggest minimal effects of neonatal deprivation and WP on latency distributions in barrel neurons of either normal or deprived rats.

In normal rats, the $36 \%$ of supragranular layer cells that fired 


\section{Latency Distributions}

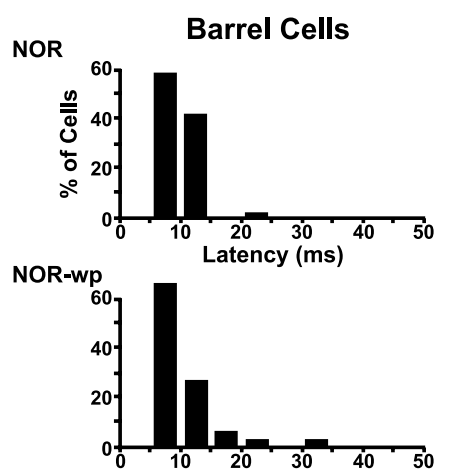

Ndep
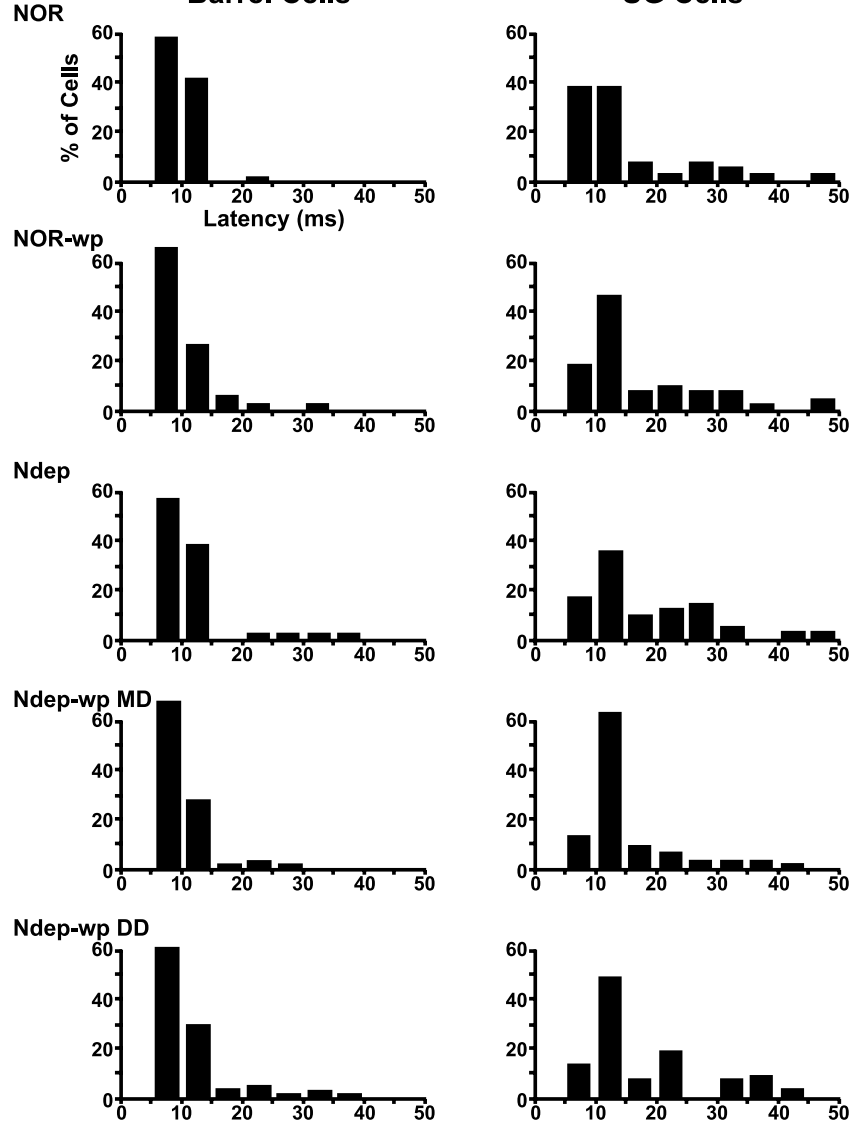

Figure 8. Distributions of modal latencies in response to the principal (D2) whisker for each of the five conditions for layer IV barrel cells (left) and for supragranular (SG) layer II and III cells (right). Cells are pooled in 5 msec bins. For nomenclature, see Figures 1 and 2.

at longer latencies rose only marginally to $37 \%$ after WP. In contrast, for neonatally deprived rats, WP produced a decrease in long-latency supragranular cells from $49 \%$ in controls (Fig. 8, NDep) to $41 \%$ and $27 \%$ for NDep-wp-DD (NDep-wp DD) and NDep-wp-MD (NDep-wp MD) rats, respectively (Fig. 8, right).

\section{Discussion}

These results confirm that abnormally low activity in sensory pathways caused by whisker trimming for approximately the first month of postnatal life results in abnormal neurotransmission through barrel field cortex, as shown by the magnitude of singleunit responses in adult rats (Simons and Land, 1987) and in juvenile rats (Glazewski and Fox, 1996; Stern et al., 2001). Plasticity induced by whisker trimming in the fully mature barrel cortex also can be demonstrated by receptive-field analysis (Diamond et al., 1993; Armstrong-James et al., 1994; Rema et al., 1998; Sachdev et al., 1998), by deoxyglucose mapping (Kossut et al., 1988; Welker et al., 1989, 1992, 1996; Kossut, 1992; Siucinska and Kossut, 1994), and by intrinsic signal-imaging techniques (Polley et al., 1999; Yazawa et al., 2001). However, there have been no equivalent previous studies on the effect of neonatal deprivation on later use-dependent plasticity of the mature barrel cortex. Our results show that activity-induced modification of cortical cell responses is significantly impaired for months after birth in the superficial layers of specific barrel columns as a result of their previous sensory deprivation during an early postnatal period of development.

Neonatal deprivation: principal whisker transmission Both anatomical (Kim and Ebner, 1999) and physiological (Armstrong-James et al., 1992; Feldman, 2000; Lubke et al., 2000; Petersen and Sakmann, 2000, 2001) evidence shows that responses of supragranular neurons in barrel columns are driven powerfully by intracolumn relay from layer IV. Spiny and aspiny stellate cells in barrels relay to supragranular neurons through profuse axonal arbors in layer II/III, projecting almost exclusively above the barrel (Kim and Ebner, 1999). Barrel projections make powerful monosynaptic connections with layer II/III cells (Feldman, 2000; Lubke et al., 2000). The same neurons collateralize in the layer IV barrel domain (Feldmeyer et al., 1999; Petersen and Sakmann, 2000). Neonatal deprivation produced by trimming only the principal D2 whisker and immediate surround D3 whisker caused neurons in layer II/III of the D2 barrel column to show a substantially reduced response to their principal whisker at maturity. In contrast, barrel neurons showed no decrease in sensory response to the neonatally deprived principal whisker input. In addition, barrel latencies to the principal whisker were virtually unaffected by deprivation. Indeed, it appears unlikely that thalamocortical transmission is compromised, because the proportion of responses occurring at the shortest latencies $(<10$ msec after stimulus) was unaltered. Therefore, deficits for the principal whisker input after neonatal deprivation appear to lie almost entirely in cortical circuitry communicating between layer IV and the supragranular layers.

Spiny stellate neurons in layer IV serve a dual role: (1) enhancement of the magnitude of principal whisker responses and (2) feedforward excitation of supragranular neurons. It is unlikely, therefore, that impaired vertical transmission is attributable only to spiny stellate neurons in layer IV, because they responded as well as normal barrel neurons at similar latencies. Therefore, we propose a deficiency in the efficacy of synaptic transmission between barrels and supragranular layers. One hypothesis that has been proposed to produce this effect depends on abnormal timing of inputs from layer IV to layer II/III that change the EPSP action potential timing in ways that induce long-term depression more readily than long-term potentiation in layer II/III cells after short periods of sensory deprivation (Feldman, 2000). The relationship linking timing-based modifications between layer IV-to-III neurotransmission and the deficits found in inhibitory neuron properties in layer IV barrels (Akhtar and Land, 1991) remains to be worked out.

In juvenile rats, plucking of all but one whisker throughout the neonatal period impairs the neuronal response to the intact whisker in supragranular layers much less than in layer IV neurons (Glazewski and Fox, 1996). Our finding of no depression in layer IV may be attributable to the more mature age at which the animals were analyzed, or it may be because we always recorded from a deprived rather than a maintained barrel column. However, the same group more recently found that if more whiskers were left intact then layer IV responses to spared whiskers were unaffected, but the supragranular neurons were still depressed, which is similar to our findings (Glazewski et al., 1998). Clearly, the timing, extent, and duration of the deprivation and the location (layer and barrel vs septum) of the cells analyzed are important variables to be addressed to differentiate the basis of these effects. 


\section{Principal whisker response plasticity}

Three days of WP at maturity failed to alter the response magnitudes to principal whisker stimulation in the barrel or supragranular layers of either neonatally deprived or control animals. In all conditions, responses were consistently larger in deprived rats than in normal controls within neonatally deprived barrels (but not supragranular layers), which is similar to the findings of Simons and Land (1987) after the partial deprivation of C-row (or all except C-row) whiskers. With neonatal whisker removal, GAD staining (Welker et al., 1989), $\mathrm{GABA}_{\mathrm{A}}$-receptor activity (Fuchs and Salazar, 1998), and GABAergic terminals on dendritic spines (Micheva and Beaulieu, 1997) decrease over several weeks in barrels of the afflicted whisker. We suggest that a fall in "infield" inhibition (Laskin and Spencer, 1979) for the whisker would develop in the deprived barrel, with disinhibition compensating for the deprivation effects.

\section{WP plasticity: surround receptive-field changes}

Insights into receptive-field plasticity require knowledge of the origins of receptive-field responses, which theoretically could be generated by both thalamocortical and intracortical circuits.

Under conditions of testing and anesthesia similar to those used in our studies, surround receptive fields (SRFs) in layers I-IV have been shown by a number of criteria to depend for their expression on intracortical column-to-column activity, initiated by principal whisker discharges (Armstrong-James and Callahan, 1991; Armstrong-James et al., 1991; Fox, 1994; Fox et al., 2001). Furthermore, WP potentiation of SRFs of barrel-column neurons in layers I-IV has been shown to be local within barrel cortex, being prevented by local suppression of intracortical transmission during WP through NMDA-receptor blockade (Rema et al., 1998). Whisker deprivation plasticity in barrel cortex is also blocked by local application of muscimol (Wallace et al., 2001), leaving thalamocortical transmission unaffected. These data support the idea that local intracortical circuitry between the principal D2 and surround D1/D3 cortical columns is critically modified by changed sensory experience.

All of our experiments compared changes in a single barrel column using various combinations of neonatal deprivation and subsequent use of the same whiskers that provide inputs to that column (principal D2 and surrounds D1 and D3). In normal adult rats, it has been established that surround inputs D1 and D3 give equivalent responses for D2 barrel columns (ArmstrongJames and Fox, 1987; Armstrong-James et al., 1991, 1992; Diamond et al., 1993; Baskerville et al., 1997; Rema et al., 1998; Sachdev et al., 1998). With 3-30 d of WP at maturity, there is a consistent shift toward the paired compared with the cut surround whisker for neurons in layers I-V (Armstrong-James et al., 1994; Diamond et al., 1994; Baskerville et al., 1997; Huang et al., 1998; Rema and Ebner, 1999). This surround bias indexes one robust form of activity-dependent synaptic plasticity in the adult cortex.

We confirmed substantial surround bias for supragranular and barrel layers in normal WP animals, and we found similar changes in the barrel neurons of the WP neonatally deprived rats. However, adult plasticity of deprived surrounds failed almost entirely for supragranular neurons in the neonatally deprived rats; no surround bias was generated when pairing the principal whisker with the neonatally trimmed surround, and only a small bias was generated for pairing with the neonatally maintained surround-row whisker.

These failures of adult plasticity could be attributable in part to a deficit in feedforward excitation from principal inputs that generate surround receptive fields in neighboring columns. However, in barrels no decrement in principal whisker response occurred with any combination of neonatal deprivation. Because receptive-field bias to WP experience also occurred readily in normal barrels, we suggest that intracortical relays constructing SRFs between adjacent columns in barrels became potentiated in response to paired whiskers in a similar manner in both neonatally deprived and normal rats.

The components of cortical circuitry responsible for reduced plasticity in supragranular layers are less apparent. Because both the neonatally maintained and the deprived SRFs were reduced in neonatally deprived rats, transmission deficits for both surrounds appear to relate to deficiencies in the intracortical input circuitry to the D2 barrel column. In this sense, all supragranularlayer circuitry of the deprived D2 column is compromised, regardless of whether it participates in generating surround or center receptive fields. One might have predicted a significantly better performance for neonatally maintained surround inputs in supragranular layers through some form of compensation. The overall effect of these reductions would be to limit the horizontal integration of whisker inputs that are a hallmark of layer III connections in the cortex. However, it is important in this context to distinguish between intactness of sensory transmission, as measured by receptive-field size, and failure of synaptic modification that requires a plasticity "challenge" to demonstrate. Either or both could be affected in complex ways by early sensory deprivation. We conclude that the main impact of deprivation must be on the intracortical circuits linking the barrel columns that fail to develop appropriately with inadequate levels of activity during the early postnatal period.

\section{References}

Akhtar ND, Land PW (1991) Activity-dependent regulation of glutamic acid decarboxylase in the rat barrel cortex: effects of neonatal versus adult sensory deprivation. J Comp Neurol 307:200-213.

Armstrong-James M (1995) The nature and plasticity of sensory processing within adult rat barrel cortex. In: Cerebral cortex, Vol I, The barrel cortex of rodents (Jones EG, Diamond IT, eds), pp 333-373. New York: Plenum.

Armstrong-James M, Callahan CA (1991) Thalamocortical processing of vibrissal information in the rat. II. Spatiotemporal convergence in the thalamic ventroposterior medial nucleus (VPm) and its relevance to generation of receptive fields of S1 cortical "barrel” neurones. J Comp Neurol 303:211-224.

Armstrong-James M, Fox K (1987) Spatiotemporal convergence and divergence in the rat SI barrel cortex. J Comp Neurol 263:265-281.

Armstrong-James M, George MJ (1988) Bilateral receptive fields of cells in rat Sm1 cortex. Exp Brain Res 70:155-165.

Armstrong-James M, Millar JM (1979) Carbon fibre microelectrodes. J Neurosci Methods 1:279-287.

Armstrong-James M, Caan AW, Fox K (1985) Threshold effects of $N$-methyl D-aspartate (NMDA) and 2-amino-phosphono valeric acid (2APV) on the spontaneous activity of neocortical single neurones in the urethane anaesthetized rat. Exp Brain Res 60:1209-1213.

Armstrong-James M, Callahan CA, Friedman MA (1991) Thalamo-cortical processing of vibrissal information in the rat. I. Intracortical origins of surround but not centre-receptive fields of layer IV neurones in the rat S1 barrel field cortex. J Comp Neurol 303:193-210.

Armstrong-James M, Fox K, Das-Gupta A (1992) Flow of excitation within rat barrel cortex on striking a single vibrissa. J Neurophysiol 68:1345-1358.

Armstrong-James M, Diamond ME, Ebner FF (1994) An innocuous bias in whisker sensation modifies receptive fields of adult rat barrel cortex neurons. J Neurosci 14:6978-6991.

Baskerville KA, Schweitzer JB, Herron P (1997) Effects of cholinergic depletion on experience-dependent plasticity in the cortex of the rat. Neuroscience 80:1159-1169.

Clark SA, Allard T, Jenkins WM, Merzenich MM (1988) Receptive fields in the body-surface map in adult cortex defined by temporally correlated inputs. Nature 332:444-445. 
Diamond ME, Armstrong-James M, Ebner FF (1993) Experience-dependent plasticity in adult rat barrel cortex. Proc Natl Acad Sci USA 90:2082-2086.

Diamond ME, Huang W, Ebner FF (1994) Laminar comparison of somatosensory cortical plasticity. Science 265:1885-1888.

Feldman DE (2000) Timing-based LTP and LTD at vertical inputs to layer II/III pyramidal cells in rat barrel cortex. Neuron 27:45-56.

Feldmeyer D, Egger V, Lubke J, Sakmann B (1999) Reliable synaptic connections between pairs of excitatory layer 4 neurones within a single "barrel" of developing rat somatosensory cortex. J Physiol (Lond) 1:169-190.

Fox K (1994) The cortical component of experience-dependent synaptic plasticity in the rat barrel cortex. J Neurosci 14:7665-7679.

Fox K, Armstrong-James M (1986) The role of the anterior intralaminar nuclei and $N$-methyl-D-aspartate receptors in the generation of spontaneous bursts in rat neocortical neurones. Exp Brain Res 63:505-518.

Fox K, Glazewski S, Wallace H (2001) The origin of surround receptive fields in barrel cortex. Soc Neurosci Abstr 27:393.

Friedberg MH, Lee SM, Ebner FF (1999) Modulation of receptive field properties of thalamic somatosensory neurons by the depth of anesthesia. J Neurophysiol 81:2243-2252.

Fuchs JL, Salazar E (1998) Effects of whisker trimming on GABA(A) receptor binding in the barrel cortex of developing and adult rats. J Comp Neurol 395:209-216.

Glazewski S, Fox K (1996) Time course of experience-dependent synaptic potentiation and depression in barrel cortex of adolescent rats. J Neurophysiol 75:1714-1729.

Glazewski S, McKenna M, Jacquin M, Fox K (1998) Experience-dependent depression of vibrissae responses in adolescent rat barrel cortex. Eur J Neurosci 10:2107-2116.

Huang W, Armstrong-James M, Rema V, Diamond ME, Ebner FF (1998) Contribution of supragranular layers to sensory processing and plasticity in adult rat barrel cortex. J Neurophysiol 80:3261-3271.

Kalaska J, Pomeranz B (1979) Chronic paw denervation causes an agedependent appearance of novel responses from forearm in "paw cortex" of kittens and adult cats. J Neurophysiol 42:618-633.

Kim U, Ebner FF (1999) Barrels and septa: separate circuits in rat barrels field cortex. J Comp Neurol 408:489-505.

Kossut M (1992) Effects of sensory deprivation upon a single cortical vibrissal column: a 2DG study. Exp Brain Res 90:639-642.

Kossut M, Hand PJ, Greenberg J, Hand CL (1988) Single vibrissal cortical column in SI cortex of rat and its alterations in neonatal and adult vibrissa-deafferented animals: a quantitative 2DG study. J Neurophysiol 60:829-852.

Laskin SE, Spencer WA (1979) Cutaneous masking. II. Geometry of excitatory and inhibitory receptive fields of single units in somatosensory cortex of the cat. J Neurophysiol 42:1061-1082.

Lubke J, Egger V, Sakmann B, Feldmeyer D (2000) Columnar organization of dendrites and axons of single and synaptically coupled excitatory spiny neurons in layer 4 of the rat barrel cortex. J Neurosci 20:5300-5311.

Merzenich MM, Kaas J, Wall J, Nelson RJ, Sur M, Felleman D (1983) Topographic reorganization of somatosensory cortical areas $3 \mathrm{~b}$ and 1 in adult monkeys following restricted deafferentation. Neuroscience 8:33-55.

Micheva KD, Beaulieu C (1997) Development and plasticity of the inhibitory neocortical circuitry with an emphasis on the rodent barrel field cortex: a review. Can J Physiol Pharmacol 75:470-478.
Petersen CC, Sakmann B (2000) The excitatory neuronal network of rat layer 4 barrel cortex. J Neurosci 20:7579-7586.

Petersen CC, Sakmann B (2001) Functionally independent columns of rat somatosensory barrel cortex revealed with voltage-dependent dye imaging. J Neurosci 21:8435-8446.

Polley DB, Chen-Bee CH, Frostig RD (1999) Two directions of plasticity in the sensory-deprived adult cortex. Neuron 24:623-637.

Rasmusson DD (1982) Reorganization of raccoon somatosensory cortex following removal of the fifth digit. J Comp Neurol 205:313-326.

Recanzone GH, Merzenich MM, Jenkins WM, Grajski KA, Dinse HR (1992a) Topographic reorganization of the hand representation in cortical area $3 \mathrm{~b}$ of owl monkeys trained in a frequency-discrimination task. J Neurophysiol 67:1031-1056.

Recanzone GH, Merzenich MM, Schreiner CE (1992b) Changes in the distributed temporal response properties of SI cortical neurons reflect improvements in performance on a temporally based tactile discrimination task. J Neurophysiol 67:1071-1091.

Rema V, Ebner FF (1999) Effect of enriched environment rearing on impairments in cortical excitability and plasticity after prenatal alcohol exposure. J Neurosci 19:10993-11006.

Rema V, Armstrong-James M, Ebner FF (1998) Experience-dependent plasticity of adult rat $\mathrm{S} 1$ cortex requires local NMDA receptor activation. J Neurosci 18:10196-10206.

Sachdev RN, Lu SM, Wiley RG, Ebner FF (1998) Role of the basal forebrain cholinergic projection in somatosensory cortical plasticity. J Neurophysiol 79:3216-3228.

Simons DJ, Land PW (1987) Early experience of tactile stimulation influences organization of somatic sensory cortex. Nature 326:694-697.

Siucinska E, Kossut M (1994) Short term changes of cortical body maps following partial vibrissectomy in adult mice. Acta Neurobiol Exp (Warsz) 54:345-354.

Stern EA, Maraval M, Svoboda K (2001) Rapid development and plasticity of layer 2/3 maps in rat barrel cortex in vivo. Neuron 31:305-315.

Wallace HS, Glazewski S, Liming K, Fox K (2001) The role of cortical activity in experience-dependent potentiation and depression of sensory responses in rat barrel cortex. J Neurosci 21:3881-3894.

Weisel TN, Hubel DH (1965) Comparison of the effects of unilateral and bilateral eye closure on cortical unit responses in kittens. J Neurophysiol 28:1029-1040.

Welker E, Soriano E, Van der Loos H (1989) Plasticity in the barrel cortex of the adult mouse: effects of peripheral deprivation on GAD-immunoreactivity. Exp Brain Res [Erratum (1989) 77:666] 74:441-452.

Welker E, Rao SB, Dorfl J, Melzer P, Van der Loos H (1992) Plasticity in the barrel cortex of the adult mouse: effects of chronic stimulation upon deoxyglucose uptake in the behaving animal. J Neurosci 12:153-170.

Welker E, Armstrong-James M, Bronchti G, Ourednik W, GheorghitaBaechler F, Dubois R, Guernsey DL, Van der Loos H, Neumann PE (1996) Altered sensory processing in the somatosensory cortex of the mouse mutant barrelless. Science 271:1864-1867.

Wong-Riley MT, Welt C (1980) Histochemical changes in cytochrome oxidase of cortical barrels after vibrissal removal in neonatal and adult mice. Proc Natl Acad Sci USA 77:2333-2337.

Yazawa I, Sasaki S, Mochida H, Kamino K, Momose-Sato Y, Sato K (2001) Developmental changes in trial-to-trial variations in whisker barrel responses studied using intrinsic optical imaging: comparison between normal and de-whiskered rats. J Neurophysiol 86:392-401. 